\title{
Proteomic identification of the SLC25A46 interactome in transgenic mice expressing SLC25A46-FLAG
}

Vasiliki-Iris Perivolidi $^{1,2}$, Foteini Violitzi ${ }^{1,2}$, Elisavet Ioannidou ${ }^{2}$, Vagelis Rinotas ${ }^{2}$, George

Stamatakis $^{2}$, Martina Samiotaki², George Panayotou ${ }^{2}$, Eleni Douni ${ }^{1,2, *}$

${ }^{1}$ Laboratory of Genetics, Department of Biotechnology, Agricultural University of

Athens, Iera Odos 75, 11855, Athens, Greece

${ }^{2}$ Institute for Bioinnovation, Biomedical Sciences Research Center "Alexander Fleming",

Fleming 34, 16672, Vari, Greece

${ }^{*}$ Corresponding author: Eleni Douni, Laboratory of Genetics, Department of

Biotechnology, Agricultural University of Athens, Iera Odos 75, 11855 Athens, Greece;

douni@aua.gr 


\section{SUPPORTING INFORMATION}

\section{Table of contents}

-Suppl. Figure S1: Rescue of thymic and splenic atrophy of Slc25a46 atc/atc mice through complementation with the SLC25A46-FLAG transgene.

-Suppl. Figure S2: Enriched SLC25A46-FLAG in the pulldown products from whole cell extracts of each tissue.

-Suppl. Figure S3: Sunburst diagram showing enriched cellular component terms of putative SLC25A46 interactors of cerebellum and cerebrum based on the synapse-specific SynGO database annotation.

-Suppl. Figure S4: Scatter plot visualization of the unsupervised PCA output regarding the samples of the four different tissues.

-Suppl. Figure S5: ATP and GTP-binding proteins identified in SLC25A46 interactomes for each tissue based on the UniProt database.

-Suppl. Figure S6: Venn diagram comparing the putative SLC25A46 protein interactors identified in TgSLC25A46-FLAG tissues with previously published interactors identified by BioID and co-IP experiments.

-Suppl. Table S1: LFQ values for proteins that co-immunoprecipitated with SLC25A46FLAG in cerebellum of Tg5537 mice identified by LC-MS/MS analysis after quantification with MaxQuant program in gene name alphabetical order.

-Suppl. Table S2: The p-value and $\log 2$ difference of proteins immunoprecipitating with SLC25A46-FLAG in cerebellum of Tg5537 mice after statistical analysis with Perseus software in difference descending order.

-Suppl. Table S3: Cellular component content of putative SLC25A46 interactors in the cerebellum of Tg5537 mice grouped in Enrichment analysis of synapse-specific SynGO database, presented in Suppl. Figure S3.

-Suppl. Table S4: LFQ values for proteins that co-immunoprecipitated with SLC25A46FLAG in cerebrum of Tg5537 mice identified by LC-MS/MS analysis after quantification with MaxQuant program in gene name alphabetical order.

-Suppl. Table S5: The p-value and $\log 2$ difference of proteins immunoprecipitating with SLC25A46-FLAG in cerebrum of Tg5537 mice after statistical analysis with Perseus software in difference descending order.

-Suppl. Table S6: Cellular component content of putative SLC25A46 interactors in the cerebrum of Tg5537 mice grouped in Enrichment analysis of synapse-specific SynGO database, presented in Suppl. Figure S3.

-Suppl. Table S7: LFQ values for proteins that co-immunoprecipitated with SLC25A46FLAG in heart of Tg5537 mice identified by LC-MS/MS analysis after quantification with MaxQuant program in gene name alphabetical order.

-Suppl. Table S8: The p-value and $\log 2$ difference of proteins immunoprecipitating with SLC25A46-FLAG in heart of Tg5537 mice after statistical analysis with Perseus software in difference descending order.

-Suppl. Table S9: LFQ values for proteins that co-immunoprecipitated with SLC25A46FLAG in thymus of Tg5537 mice identified by LC-MS/MS analysis after quantification with MaxQuant program in gene name alphabetical order.

-Suppl. Table S10: The p-value and $\log 2$ difference of proteins immunoprecipitating with SLC25A46-FLAG in thymus of Tg5537 mice after statistical analysis with Perseus software in difference descending order. 
-Suppl. Table S11: Biological process content of putative SLC25A46 interactors in the cerebellum of Tg5537 mice grouped in Enrichment analysis of Cytoscape using the ClueGO/CluePedia plugins.

-Suppl. Table S12: Biological process content of putative SLC25A46 interactors in the cerebrum of Tg5537 mice grouped in Enrichment analysis of Cytoscape using the ClueGO/CluePedia plugins.

-Suppl. Table S13: Biological process content of putative SLC25A46 interactors in the heart of Tg5537 mice grouped in Enrichment analysis of Cytoscape using the ClueGO/CluePedia plugins.

-Suppl. Table S14: Biological process content of putative SLC25A46 interactors in the thymus of Tg5537 mice grouped in Enrichment analysis of Cytoscape using the ClueGO/CluePedia plugins.

-Suppl. Table S15: Frequency of reports in the FLAG CRAPome repository for the identified SLC25A46 interactors of this study.

-Suppl. Table S16: Biological process content of putative SLC25A46 interactors common in two or more tissues grouped in Enrichment analysis of Cytoscape using the ClueGO/CluePedia plugins. 


\section{Supplementary Figure S1}

A

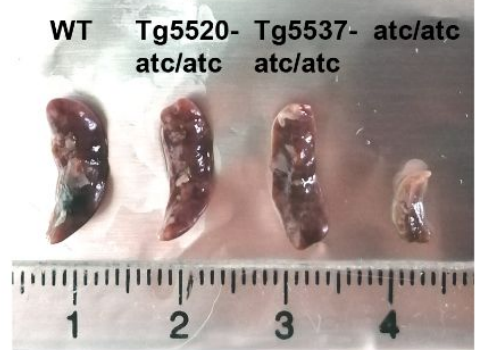

B

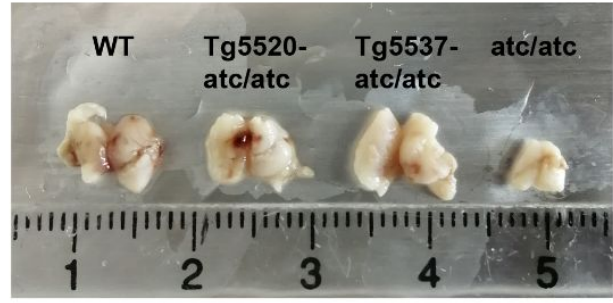

Supplementary Figure S1: Rescue of thymic and splenic atrophy of Slc $25 \mathrm{a} 46^{\text {atc/atc }}$ mice through complementation with the SLC25A46-FLAG transgene. Representative photographs of (A) spleens and (B) thymi dissected from WT, Tg5520/Slc25a46 atc/atc, $\operatorname{Tg} 5537 / \operatorname{Slc} 25 \mathrm{a} 46^{\text {atc/atc }}$ and Slc25a46 atc/atc mice (atc/atc). 


\section{Supplementary Figure S2}

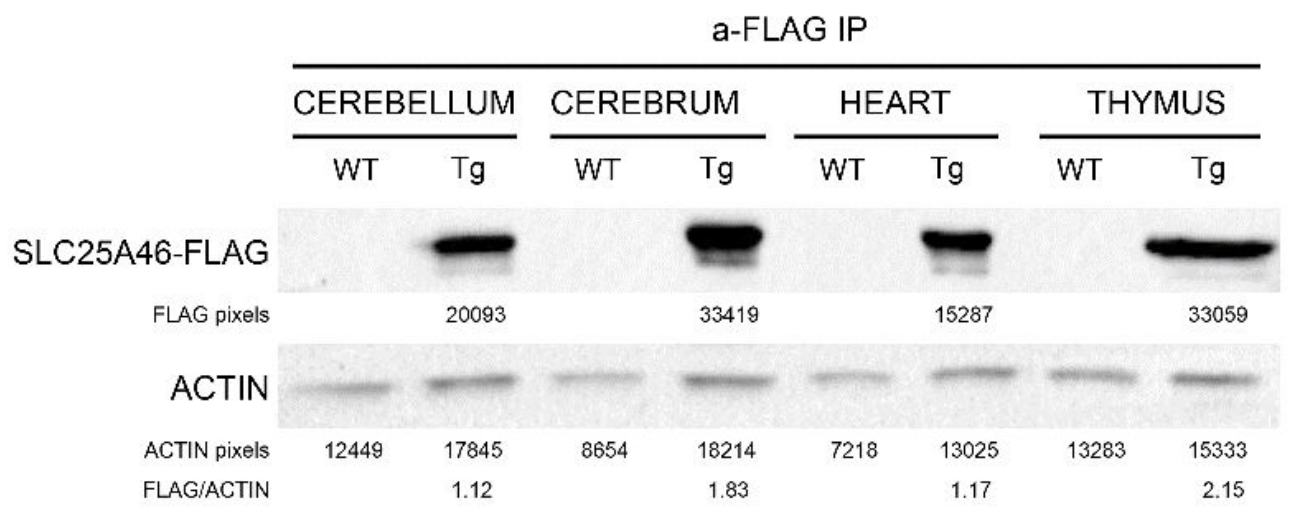

Supplementary Figure S2: Enriched SLC25A46-FLAG in the pulldown products from whole cell extracts of each tissue. Western blot identified SLC25A46-FLAG in SLC25A46-FLAG-IP products in cerebellum, cerebrum, heart, and thymus using an antibody against FLAG. IPs derived from equal input quantities from transgenic and WT tissues. ACTIN was used as internal control since it has been shown to bind weakly to the FLAG-agarose beads according to CRAPome repository. Numbers below each western blot indicate pixel quantification, while the ratio of FLAG to ACTIN (FLAG/ACTIN) pixels is also shown. 


\section{Supplementary Figure S3}
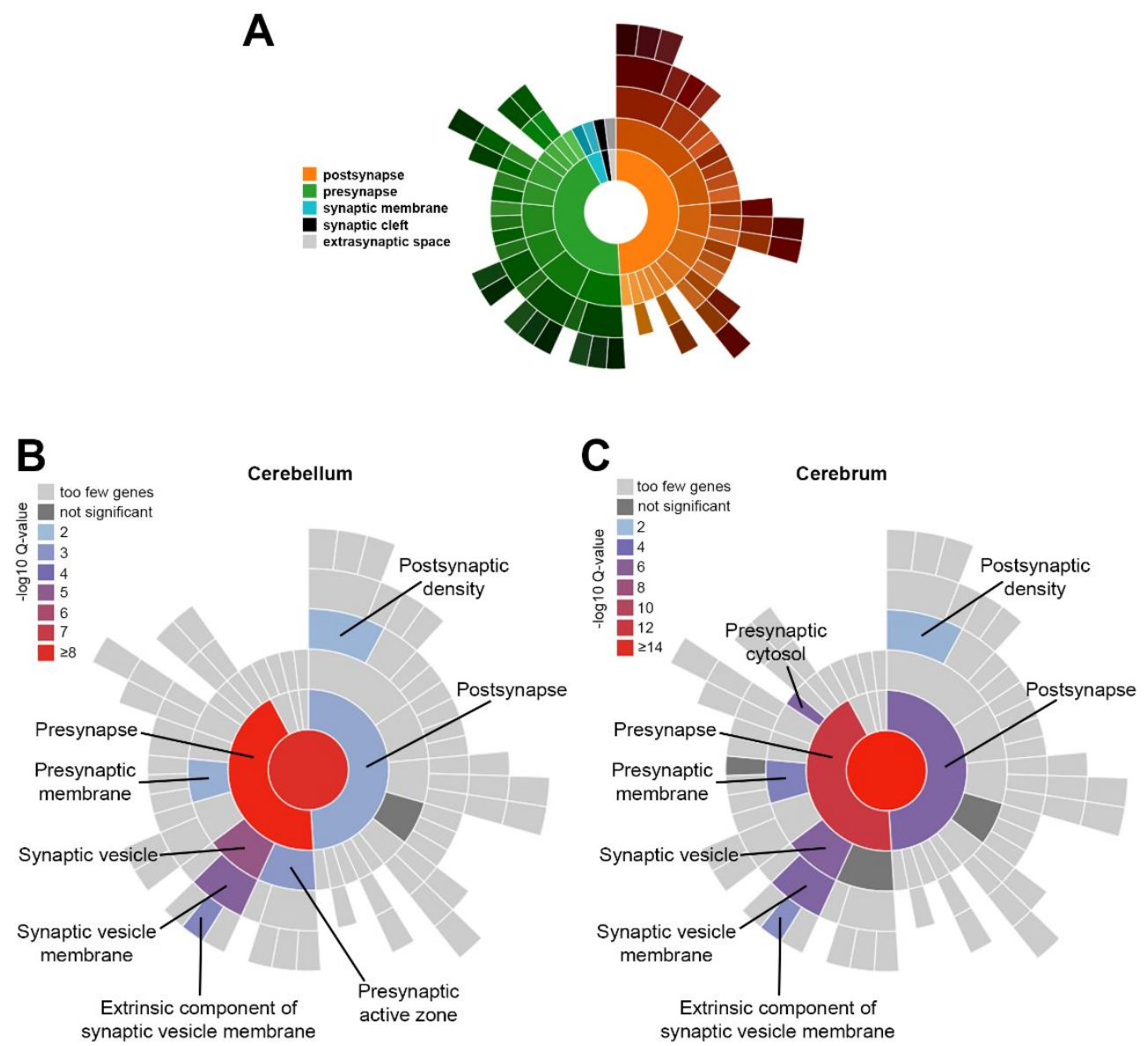

Supplementary Figure S3: Sunburst plot showing enriched cellular component terms of putative SLC25A46 interactors of (B) cerebellum and (C) cerebrum based on the synapsespecific SynGO database annotation. (A) Sunburst plot represents synaptic annotated GO cellular component ontologies. The color encodes the significance of the enrichment ($\log 10$ (Q-value)). Full list of enriched biological functions is shown in Suppl. Tables S3 and S6. 


\section{Supplementary Figure S4}

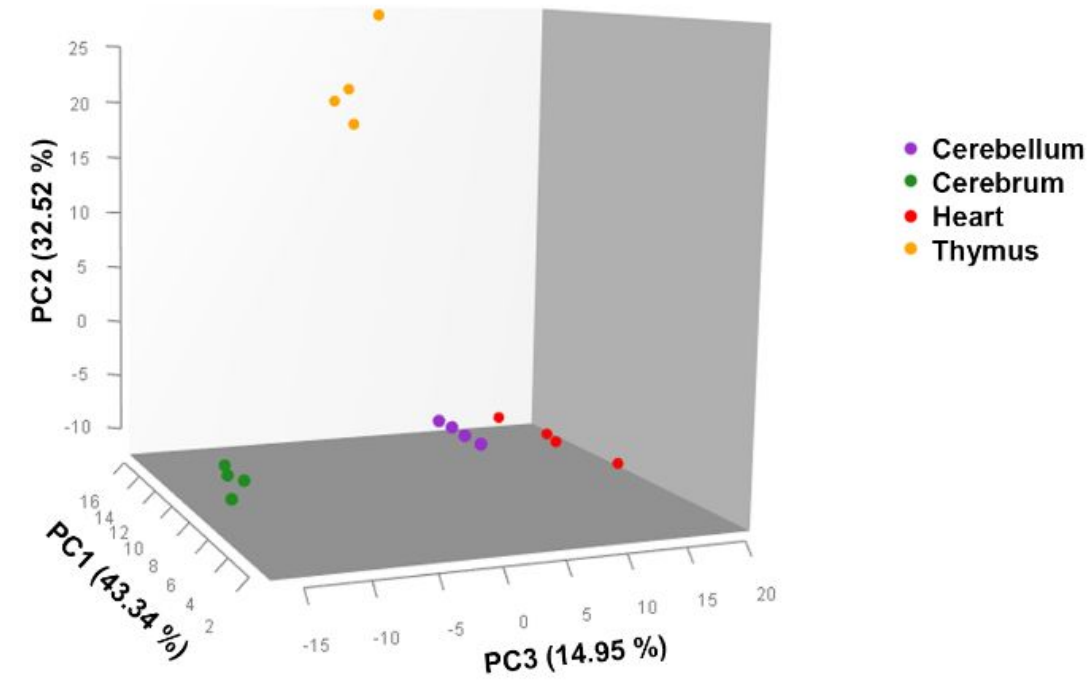

Supplementary Figure S4: Scatter plot visualization of the unsupervised PCA output regarding the samples of the four different tissues (Cerebellum, Cerebrum, Heart and Thymus). The plot demonstrates the distribution of the samples in the rotated space and their segregation according to the tissue they came from. 


\section{Supplementary Figure S5}

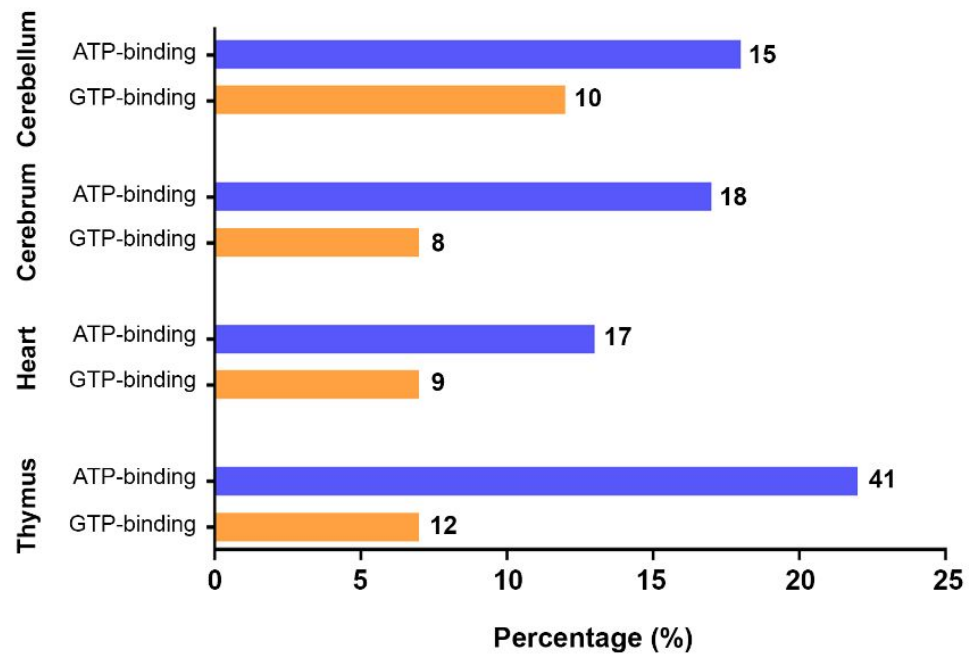

Supplementary Figure S5: ATP and GTP-binding proteins identified in SLC25A46 interactomes for each tissue based on the UniProt database. The percentage of the SLC25A46 interacting proteins in each category was calculated and plotted. 


\section{Supplementary Figure S6}

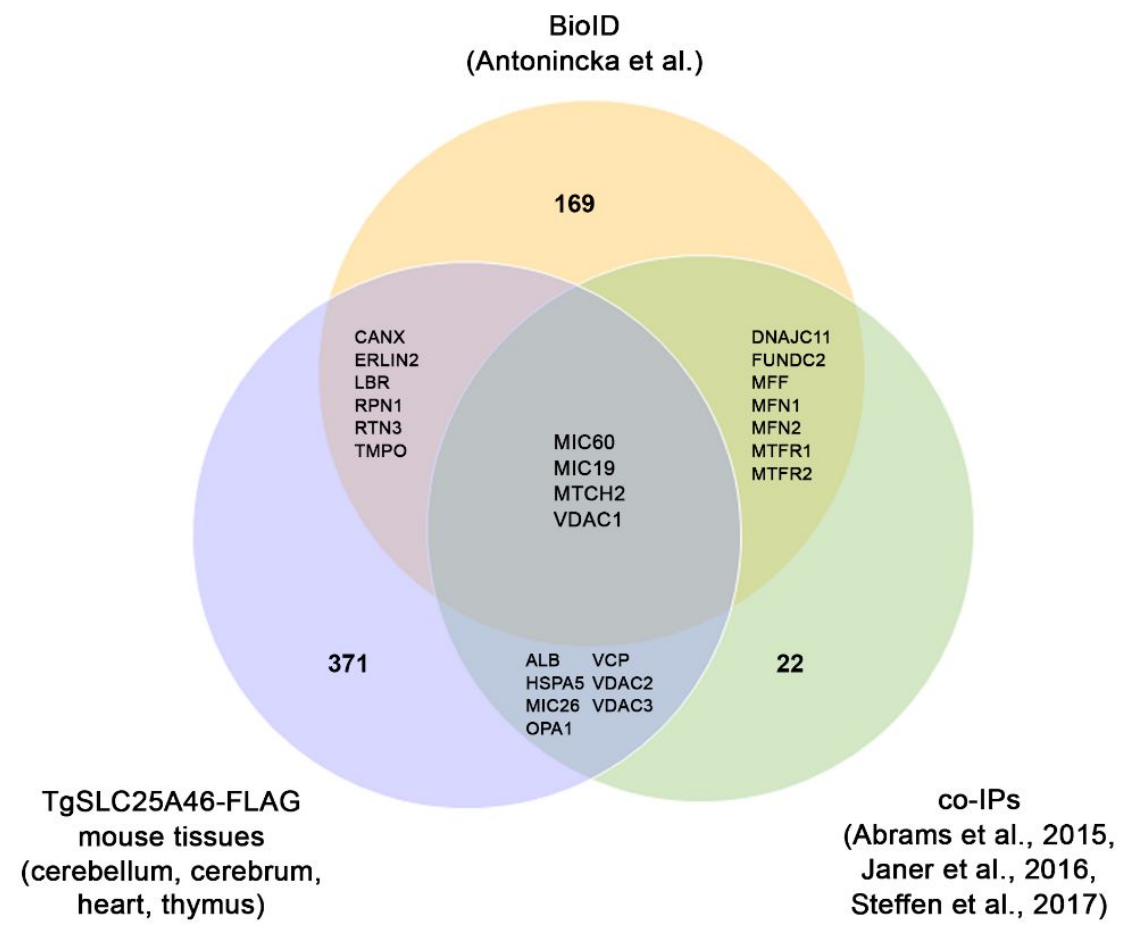

Supplementary Figure S6: Venn diagram comparing the putative SLC25A46 protein interactors identified in TgSLC25A46-FLAG tissues with previously published interactors identified by BioID and co-IP experiments. 